\title{
Genetic variants in the ZNF208 gene are associated with esophageal cancer in a Chinese Han population
}

\author{
Huijie Wang ${ }^{1}$, Jianzhong Yu ${ }^{2}$, Yanling Guo ${ }^{1}$, Zhengxing Zhang ${ }^{1}$, Guoqi Liu ${ }^{1}$, \\ Jingjie $\mathrm{Li}^{3}$, Xiyang Zhang ${ }^{4}$, Tianbo Jin ${ }^{3}$, Zhaoxia Wang ${ }^{1}$ \\ ${ }^{1}$ Department of Intergrated Traditional Chinese and Western Medicine in Oncology, Affiliated Luoyang Central Hospital, \\ Zhengzhou University, Luoyang 471000, China \\ ${ }^{2}$ Department of Neurology, Haikou People's Hospital, Haikou 570208, Hainan, China \\ ${ }^{3}$ Key Laboratory of Resource Biology and Biotechnology in Western China, Ministry of Education, School of Life Sciences, \\ Northwest University, Xi'an, Shaanxi 710069, China \\ ${ }^{4} X i$ 'an Tiangen Precision Medical Institute, Xi'an, Shaanxi 710075, China \\ Correspondence to: Zhaoxia Wang, email: wangzhaoxia_a@163.com \\ Keywords: ZNF208, esophageal cancer, single nucleotide polymorphisms (SNPs), association study
}

Received: July 05, 2016

Accepted: October 29, 2016

Published: November 19, 2016

\section{ABSTRACT}

Previous studies showed an association between the ZNF208 gene and gastric cancer. In this study, we investigated the association between single nucleotide polymorphisms (SNPs) in ZNF208 and the risk of esophageal cancer in a Chinese Han population. We conducted a case-control study that included 386 cases and 495 controls. Five SNPs were selected from previous genome-wide association studies and genotyped using the Sequenom MassARRAY platform. Unconditional logistic regression was used to calculate odds ratios and $95 \%$ confidence intervals after adjustment for age and gender. Logistic regressionl analysis showed that two SNPs (rs8103163 and rs7248488) were associated with an increased risk of esophageal cancer under different inheritance models after Bonferroni correction. Haplotype analysis suggested that the four variants comprised one block, and that the $G_{r s 2188972}$ $\mathrm{C}_{\mathrm{rs2188971}} \mathrm{C}_{\mathrm{rs8103163}} \mathrm{C}_{\mathrm{rs7248488}}$ haplotype was significantly correlated with an increased risk of esophageal cancer. Our data indicate that variants in ZNF208 are contribute to the susceptibility to esophageal cancer in a Chinese Han population.

\section{INTRODUCTION}

Esophageal carcinoma is the sixth leading cause of cancer-related mortality worldwide [1]. Previous studies have demonstrated that China has the highest incidence of this aggressive malignancy [2]. The incidence rate was $186.34 / 10^{5}$ person-years in China [3] compared to $8.34 / 10^{5}$ person-years among Caucasian men in the U.S. [1]. The main risk factors for esophageal cancer are gastroesophageal reflux disease, smoking, and obesity [47]. However, only a subset of individuals who are exposed to these environmental risk factors actually develop esophageal cancer, suggesting that others factors (e.g. heritable) may also impact susceptibility.

Single nucleotide polymorphisms (SNPs) that are correlated with an increased risk of esophageal cancer have been identified previously [8-10]. The Zinc finger protein 208 (ZNF208) gene is located in the p12 region of human chromosome 19. ZNF208 is a member of the Zinc finger family of proteins, which bind to DNA through a series of zinc finger motifs and regulate gene transcription [11, 12]. Mutations in ZNF208 have been observed in gastric cancer. Thus, it may act as a tumor suppressor [13]. This gene was also associated with the response to imatinib mesylate treatment in patients with gastrointestinal stromal tumor [14]. Recently, an association between one variant (rs8105767) within the ZNF208 gene and telomere length was identified in a genome-wide association study. Interestingly, r s8105767 was associated with the risk of neuroblastoma, but not osteosarcoma or leukemia [15]. This SNP was also associated with the risk of lung adenocarcinoma, but not colorectal, breast, or prostate cancer [16]. It was also not associated with chronic lymphocytic leukemia or glioma $[17,18]$. This variant was associated with reduced telomere length and coronary artery disease in a European population [19]. However, this SNP was not associated with reduced telomere length in squamous cell carcinoma of the head and neck in a Chinese 
Table 1: Allele frequencies in cases and controls and odds ratio estimates for esophageal cancer

\begin{tabular}{|c|c|c|c|c|c|c|c|c|c|}
\hline \multirow{2}{*}{ SNP_ID } & \multirow{2}{*}{ Position } & \multirow{2}{*}{ Location } & \multirow{2}{*}{ Allele $A^{\text {a }} / \mathbf{B}$} & \multicolumn{2}{|c|}{ MAF } & \multirow{2}{*}{$\begin{array}{c}\text { HWE } \\
p \text {-value }\end{array}$} & \multirow{2}{*}{ ORs } & \multirow{2}{*}{$95 \% \mathrm{CI}$} & \multirow{2}{*}{$p$-value } \\
\hline & & & & Case & Control & & & & \\
\hline rs2188972 & 22149458 & 3'utr & $\mathrm{A} / \mathrm{G}$ & 0.52 & 0.49 & 1 & 1.1 & $0.91-1.32$ & 0.342 \\
\hline rs2188971 & 22152182 & 3'utr & $\mathrm{T} / \mathrm{C}$ & 0.32 & 0.3 & 0.593 & 1.08 & $0.88-1.32$ & 0.488 \\
\hline rs8103163 & 22174752 & intron & $\mathrm{A} / \mathrm{C}$ & 0.33 & 0.3 & 0.671 & 1.13 & $0.92-1.38$ & 0.254 \\
\hline rs 7248488 & 22188709 & intron & $\mathrm{A} / \mathrm{C}$ & 0.33 & 0.3 & 0.595 & 1.13 & $0.92-1.38$ & 0.261 \\
\hline rs8105767 & 22215441 & intron & $\mathrm{G} / \mathrm{A}$ & 0.29 & 0.299 & 0.237 & 0.95 & $0.77-1.17$ & 0.644 \\
\hline
\end{tabular}

MAF, minor allele frequency; HWE: Hardy-Weinberg Equilibrium; ORs, odds ratios; CI, confidence interval.

a, Minor allele.

Han population [20], and it was also not associated with gastric cancer susceptibility [21].

We performed a case-control study to evaluate the association between SNPs/haplotypes in the ZNF208 gene and esophageal cancer susceptibility in a Chinese Han population. Fivevariants (rs2188972, rs2188971, rs8103163, rs7248488 and rs8105767) were included in the analysis. Our data. provide evidence for a correlation between SNPs in the ZNF208 gene and esophageal cancer in the Chinese Han population.

\section{RESULTS}

\section{Characteristics of the study population}

We enrolled 385 esophageal cancer patients and 495 healthy controls in the study. The mean age was $60.68 \pm$ 8.95 years in the case group and $54.48 \pm 9.44$ years in the control group. There were 300 men (79.8\%) and 78 women $(20.2 \%)$ in the case group, and 180 men (36.4\%) and 315 women $(63.6 \%)$ in the control group. There were no significant differences in age and gender between the cases and the controls $(p<0.001)$.

\section{Associations between the five SNPs in ZNF208 and the risk of esophageal cancer}

The five SNPs that were genotyped are shown in Table 1. All SNPs were in Hardy-Weinberg equilibrium (HWE) in the control samples. The SNP distribution frequencies were calculated according to the genotyping data for the case and control groups. The minor allele frequencies are also shown in Table 1. All the alleles of the SNPs had non-significant Chi-square $(\chi 2)$ values.

We identified associations between four SNPs in ZNF208 and esophageal cancer using a logistic regression model after adjustment for age and gender. The detailed genotype distributions under various models are presented in Table 2. Interestingly, rs2188972 was associated with an increased risk of esophageal cancer in homozygote $(p=0.046)$ and additive $(p=0.046)$ models. Additionally, rs2188971 demonstrated a significant association in dominant $(p=0.037)$ and additive $(p=0.034)$ models. Rs8103163 showed a significant association in dominant $(p=0.009)$, additive $(p=0.008)$, and co-dominant models (homozygote $p=0.037$, heterozygote $p=0.024$ ). A significant association was also detected between rs7248488 and the risk of esophageal cancer in dominant $(p=0.017)$ and additive $(p=0.01)$ models, as well as in the co-dominant model for the homozygous genotype $(p=0.028)$. We did not observe any association between rs8105767 and esophageal cancer risk in any of the models. Only two mutations, rs8103163 (dominant $p=0.045$; additive $p=0.040$ ) and rs7248488 (additive $p=0.050$ ), were associated with an increased risk of esophageal cancer after Bonferroni correction.

\section{Association between haplotypes in ZNF208 and esophageal cancer}

We performed linkage disequilibrium analysis for the five SNPs in the logistic regression model. One block was detected based on the calculated D' values (Figure 1). Three common haplotypes are shown in Table 3. We found that the $\mathrm{A}_{\mathrm{rs} 2188972} \mathrm{~T}_{\mathrm{rs} 2188971} \mathrm{~A}_{\mathrm{rs} 8103163} \mathrm{~A}_{\mathrm{rs} 7248488}$ haplotype was associated with a significantly increased risk of esophageal cancer (odds ratio $[\mathrm{OR}]=1.33,95 \%$ confidence interval [CI] 1.05-2.38, $p=0.031)$. An additional haplotype, $\mathrm{G}_{\mathrm{rs} 2188972} \mathrm{C}_{\mathrm{rs} 2188971} \mathrm{C}_{\mathrm{rs} 8103163} \mathrm{C}_{\mathrm{rs} 7248488}$, which consisted of the major allele of all four SNPs, reached statistical significance $(p=0.031, \mathrm{OR}=0.79,95 \%$ CI $0.64-0.98)$. Interestingly, this haplotype had a protective effect against esophageal cancer.

\section{DISCUSSION}

We assessed the relationship between SNPs in the ZNF208 gene and esophageal cancer in a Chinese Han population. Our results indicate that four SNPs (rs2188972, rs2188971, rs8103163, and rs7248488) are associated with an increased risk of esophageal cancer after adjustment for age and gender. However, we did not 
Table 2: Logistic regression analysis of the association between SNPs and esophageal cancer risk

\begin{tabular}{|c|c|c|c|c|c|c|}
\hline SNP & Model & Genotype & Case & Control & OR(95\%CI) & $P^{*}$ value \\
\hline \multirow[t]{8}{*}{ rs2188972 } & Co-dominat & GG & 92 & 129 & & \\
\hline & Heterozygote & $\mathrm{AG}$ & 192 & 248 & $1.25(0.87-1.81)$ & 0.232 \\
\hline & Homozygote & AA & 101 & 118 & $1.55(1.01-2.37)$ & 0.046 \\
\hline & Dominant & GG & 92 & 129 & & \\
\hline & & AG-AA & 293 & 366 & $1.34(0.95-1.90)$ & 0.097 \\
\hline & Recessive & AG-GG & 284 & 377 & & \\
\hline & & AA & 101 & 118 & $1.33(0.94-1.89)$ & 0.108 \\
\hline & Log-additive & & & & $1.24(1.00-1.54$ & 0.046 \\
\hline \multirow[t]{8}{*}{ rs2188971 } & Co-dominat & $\mathrm{CC}$ & 179 & 243 & & \\
\hline & Heterozygote & $\mathrm{TC}$ & 163 & 202 & $1.35(0.98-1.86)$ & 0.069 \\
\hline & Homozygote & $\mathrm{TT}$ & 39 & 47 & $1.57(0.92-2.68)$ & 0.101 \\
\hline & Dominant & $\mathrm{CC}$ & 179 & 243 & & \\
\hline & & TC-TT & 202 & 249 & $1.39(1.02-1.88)$ & 0.037 \\
\hline & Recessive & TC-CC & 342 & 445 & & \\
\hline & & $\mathrm{TT}$ & 39 & 47 & $1.36(0.81-2.27)$ & 0.24 \\
\hline & Log-additive & & & & $1.29(1.02-1.63)$ & 0.034 \\
\hline \multirow[t]{8}{*}{ rs8103163 } & Co-dominat & $\mathrm{CC}$ & 170 & 243 & & \\
\hline & Heterozygote & $\mathrm{AC}$ & 167 & 205 & $1.45(1.05-2.01)$ & 0.024 \\
\hline & Homozygote & AA & 40 & 47 & $1.77(1.03-3.03)$ & 0.037 \\
\hline & Dominant & $\mathrm{CC}$ & 170 & 243 & & \\
\hline & & $\mathrm{AC}+\mathrm{AA}$ & 207 & 252 & $1.51(1.11-2.05)$ & 0.009 \\
\hline & Recessive & $\mathrm{AC}+\mathrm{CC}$ & 337 & 448 & & \\
\hline & & AA & 40 & 47 & $1.48(0.89-2.46)$ & 0.135 \\
\hline & Log-additive & & & & $1.37(1.09-1.74)$ & 0.008 \\
\hline \multirow[t]{8}{*}{ rs 7248488} & Co-dominat & $\mathrm{CC}$ & 175 & 243 & & \\
\hline & Heterozygote & $\mathrm{AC}$ & 166 & 204 & $1.38(0.99-1.90)$ & 0.0503 \\
\hline & Homozygote & AA & 43 & 48 & $1.8(1.06-3.04)$ & 0.028 \\
\hline & Dominant & $\mathrm{CC}$ & 175 & 243 & & \\
\hline & & $\mathrm{AC}+\mathrm{AA}$ & 209 & 252 & $1.45(1.07-1.97)$ & 0.017 \\
\hline & Recessive & $\mathrm{AC}+\mathrm{CC}$ & 341 & 447 & & \\
\hline & & AA & 43 & 48 & $1.54(0.93-2.54)$ & 0.09 \\
\hline & Log-additive & & & & $1.36(1.08-1.71)$ & 0.01 \\
\hline \multirow[t]{8}{*}{ rs8105767 } & Co-dominat & AA & 195 & 236 & & \\
\hline & Heterozygote & GA & 156 & 219 & $0.89(0.65-1.21)$ & 0.45 \\
\hline & Homozygote & GG & 33 & 38 & $1.44(0.81-2.58)$ & 0.218 \\
\hline & Dominant & AA & 195 & 236 & & \\
\hline & & $\mathrm{GA}+\mathrm{GG}$ & 189 & 257 & $0.95(0.71-1.29)$ & 0.76 \\
\hline & Recessive & $\mathrm{GA}+\mathrm{AA}$ & 351 & 455 & & \\
\hline & & GG & 33 & 38 & $1.53(0.87-2.68)$ & 0.142 \\
\hline & Log-additive & & & & $1.05(0.82-1.33)$ & 0.705 \\
\hline
\end{tabular}

*Adjusted for age and gender. 
detect an association between rs8105767 and esophageal cancer susceptibility. This SNP was previously shown to be associated with reduced telomere length in a European population [19]. Several studies have provided evidence that telomere shortening increases the risk of esophageal carcinoma [10, 22-24]. However, rs8105767 showed no significant association with telomere length or gastric cancer in a Chinese population [21, 25], and it was not associated with esophageal cancer in our study. Thus, this SNP may not affect telomere length. Alternatively, the results may be attributed to differences in genetic background between the Chinese Han and European populations.

Zinc finger proteins are the largest family of transcriptional regulators. These proteins have critical roles in cell differentiation and development. There is evidence that Zinc finger proteins are broadly involved in tumorigenesis [26, 27]. Wang et al. [26] showed that ZNF300 promoted carcinogenesis by modulating the NF- $\gamma \mathrm{B}$ pathway. Additionally, overexpression of ZNF300 stimulated cancer cell proliferation in vitro and significantly enhanced tumor development and metastasis in a xenograft mouse model. Another study demonstrated that ZNF280B promotes prostate cancer cell growth and survival through down-regulation of p53 [28]. Wang et al. determined that the ZNF motif (Cys2-His2) in ZNF425 was the main region responsible for the transcriptional repression activity of this protein [29].

The conserved $\mathrm{C} 2 \mathrm{H} 2$ motif is also present in ZNF208, suggesting that it may be involved in cancer development and progression. Rink et al. [14] found that the expression of the ZNF43, ZNF208, and ZNF91 genes was correlated with the response to imatinib mesylate in patients with gastrointestinal stromal tumor. Depletion of any of the genes could alter treatment response. In another study of gastric cancer [13], the activity and mRNA expression of ZNF208 were reduced as a consequence of somatic mutations, suggesting ZNF208 has a central role in tumor suppression. In our study, rs2188972, rs2188971, rs8103163, and rs 7248488 were associated with the risk of esophageal cancer. All four SNPs are either intronic or 3' UTR variants, which do not alter the coding sequence of the protein (Table 2). However, these SNPs may regulate transcription and affect gene expression.

Given the limited value of analysis of a single SNP, we evaluated the association between haplotypes in the ZNF208 gene and esophageal cancer susceptibility. The ATAA haplotype accounted for $32.15 \%$ (case group) and $30.1 \%$ (control group) of all possible marker combinations (Table 3). After adjustment for gender and age, the results

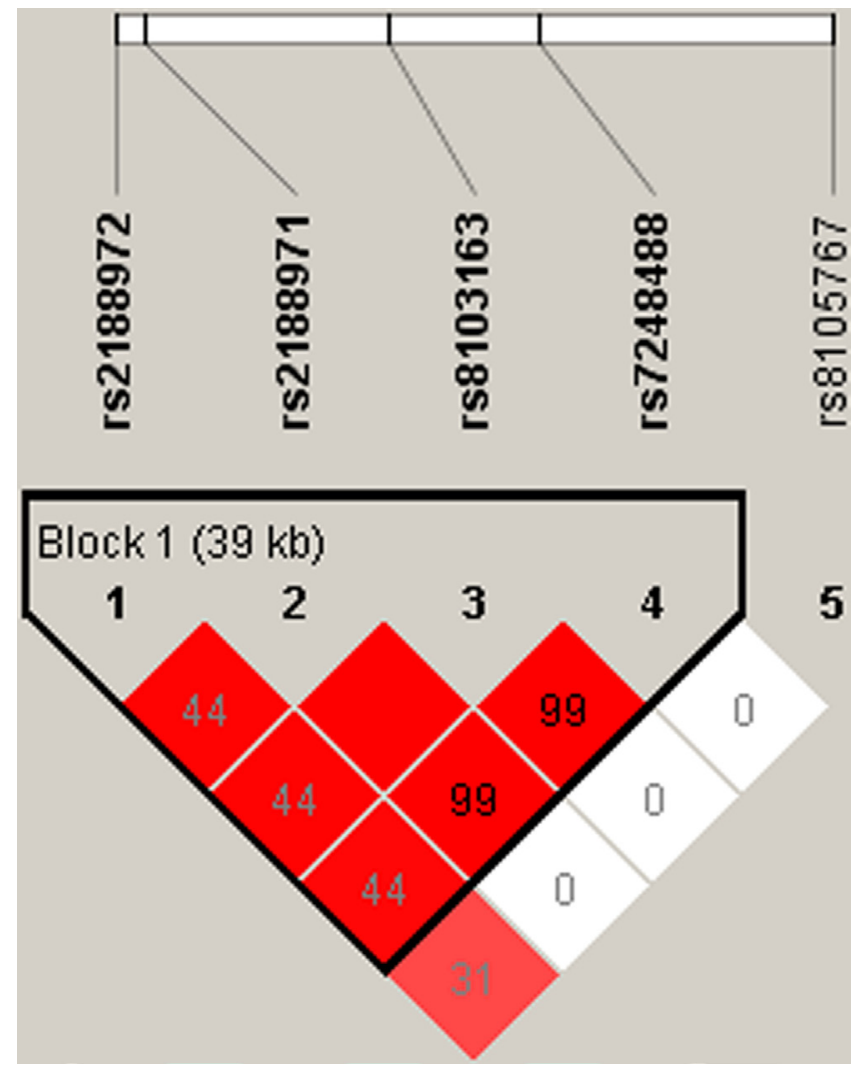

Figure 1: Linkage disequilibrium (LD) plots containing five SNPs from ZNF208. 
Table 3: The haplotype frequencies of $Z N F 208$ polymorphisms and esophageal cancer risk

\begin{tabular}{lccccccc}
\hline rs2188972 & rs2188971 & rs8103163 & rs7248488 & Case\% & Control\% & OR(95\%CI) & $\boldsymbol{p}^{*}$ value \\
\hline $\mathrm{G}$ & $\mathrm{C}$ & $\mathrm{C}$ & $\mathrm{C}$ & 48.56 & 50.91 & $0.79(0.64-0.98)$ & $\mathbf{0 . 0 3 1}$ \\
$\mathrm{A}$ & $\mathrm{C}$ & $\mathrm{C}$ & $\mathrm{C}$ & 18.64 & 18.79 & $0.94(0.71-1.25)$ & 0.69 \\
$\mathrm{~A}$ & $\mathrm{~T}$ & $\mathrm{~A}$ & $\mathrm{~A}$ & 32.15 & 30.1 & $1.33(1.05-2.38)$ & $\mathbf{0 . 0 1 8}$ \\
\hline
\end{tabular}

*Adjusted for age and gender.

showed that the ATAA haplotype was associated with a significantly increased risk of esophageal cancer.

Although our study has provided evidence for an association between genetic variants in $Z N F 208$ and esophageal cancer, the results should be interpreted with caution. A post-hoc power analysis showed that all of the SNPs failed to reach the minimum level of statistical power $(75 \%)$ for a case-control study. Thus, although the differences difference were statistically significant, the results must be verified in studies with larger sample sizessize. Our study had several limitations. First, selection bias and the limited sample size may have increased the number of false positive associations. Second, many other risk factors for esophageal cancer (e.g., smoking, alcohol consumption, pathology) were not included in the analysis. Finally, we did not analyze the biological functions of the mutations, which should be investigated in future studies.

In conclusion, our data indicate that rs2188972, rs2188971, rs8103163, and rs7248488 in the ZNF208 gene as well as the ATAA haplotype are associated with an increased risk of esophageal cancer in a Chinese Han population.

\section{MATERIALS AND METHODS}

\section{Study population and sample collection}

Patient data and venous blood samples were collected starting in May 2014 at the First Affiliated Hospital of Xi' an Jiaotong University. A total of 386 cases and 495 controls were enrolled in our study. The case group consisted of inpatients who were diagnosed with pathologically verified esophageal cancer. All subjects participated in the screening program by undergoing an endoscopic staining examination with $1.2 \%$ iodine solution, and biopsies were collected from unstained areas of the mucosa. Tissue sections were reviewed by two experienced pathologists in order to ensure tumor cell purity $>50 \%$ and confirm the histological type. None of the patients had a history of other cancers or had received chemotherapy or radiotherapy. Patients who had comorbidities such as diabetes mellitus, hypertension, or any endocrine disorders were excluded. The controls were cancer-free individuals recruited from the health checkup center at the same hospital. Patient demographic data were also collected. All subjects were genetically unrelated Han Chinese and local residents of Northwest China.

The study was approved by the Clinical Research Ethics of Northwest University and Xi'an Jiaotong University. Written informed consent was obtained from all participants. Venous blood samples $(5 \mathrm{~mL})$ were collected from each subject into tubes containing EDTA, centrifuged, and stored at $-80^{\circ} \mathrm{C}$.

\section{Genotyping}

Few studies have investigatedinvestigate the associations between SNPs in the ZNF208 and esophageal cancer. We searched Pubmed and the Hapmap database for ZNF208 SNPs with a MAF $>0.05$ in the Chinese Han population. Five SNPs (rs2188972, rs2188971, rs8103163, rs7248488, and rs8105767) were selected for our study. Genomic DNA was extracted from the whole blood samples using the GoldMag-Mini Purification Kit (GoldMag Co. Ltd. Xian, China) according to the manufacturer's instructions. DNA concentrations were measured using a NanoDrop 2000 (Thermo Scientific, Waltham, Massachusetts, USA). SNPs were genotyped using the Sequenom MassARRAY RS1000 and the manufacturer's protocol [30]. The Sequenom MassARRAY Assay Design 3.0 software was used to design a multiplexed SNP MassEXTEND assay [31]. The Sequenom Typer 3.0 Software (Sequenom, Inc) was used for data management and analyses [30, 31].

\section{Statistical analysis}

Genotype frequencies in the control subjects were tested for departure from HWE using $\chi 2$ tests. The genotype and allele distributions in the esophageal cancer patients and control subjects were compared using $\chi 2$ tests. ORs and $95 \%$ CIs were calculated by unconditional logistic regression analysis in co-dominant, additive, recessive, and dominant models after adjustment for confounding factors such as gender and age [32]. A twosided $p<0.05$ was considered statistically significant. The statistical analyses above were performed using Microsoft Excel and SPSS 20.0 (SPSS Inc., Chicago, IL, USA). Linkage disequilibrium and haplotypes were analyzed with the SHEsis software [33]. The Sampsize platform 
(http://sampsize.sourceforge.net/iface/s3.html) was used to evaluate the statistical power of this case-control study.

\section{CONFLICTS OF INTEREST}

The authors declare no conflicts of interest.

\section{REFERENCES}

1. Pennathur A, Gibson MK, Jobe BA, Luketich JD. Oesophageal carcinoma. The Lancet. 2013; 381:400-412.

2. Ke L. Mortality and incidence trends from esophagus cancer in selected geographic areas of China circa 1970-90. Int J Cancer. 2002; 102:271-274.

3. Chen W, Zheng R, Zeng H, Zhang S, He J. Annual report on status of cancer in China, 2011. Chin J Cancer Res. 2015; 27:2-12.

4. Rustgi AK, El-Serag HB. Esophageal carcinoma. N Engl J Med. 2014; 371:2499-2509.

5. Ohashi S, Miyamoto S, Kikuchi O, Goto T, Amanuma Y, Muto M. Recent Advances From Basic and Clinical Studies of Esophageal Squamous Cell Carcinoma. Gastroenterology. 2015; 149:1700-1715.

6. Domper Arnal MJ, Ferrandez Arenas A, Lanas Arbeloa A. Esophageal cancer: Risk factors, screening and endoscopic treatment in Western and Eastern countries. World J Gastroenterol. 2015; 21:7933-7943.

7. Schneider JL, Corley DA. A review of the epidemiology of Barrett's oesophagus and oesophageal adenocarcinoma. Best Pract Res Clin Gastroenterol. 2015; 29:29-39.

8. Wu C, Wang Z, Song X, Feng XS, Abnet CC, He J, Hu N, Zuo XB, Tan W, Zhan Q, Hu Z, He Z, Jia W, et al. Joint analysis of three genome-wide association studies of esophageal squamous cell carcinoma in Chinese populations. Nat Genet. 2014; 46:1001-1006.

9. Yin J, Wang L, Zheng L, Wang X, Shi Y, Shao A, Ding G, Liu C, Chen S, Tang W, Gu H. TERT-CLPTM1L Rs401681 $\mathrm{C}>\mathrm{T}$ polymorphism was associated with a decreased risk of esophageal cancer in a Chinese population. PloS one. 2014; 9:e100667.

10. Zhang Y, Calado R, Rao M, Hong JA, Meeker AK, Dumitriu B, Atay S, McCormick PJ, Garfield SH, Wangsa D, PadillaNash HM, Burkett S, Zhang M, et al. Telomerase variant A279T induces telomere dysfunction and inhibits noncanonical telomerase activity in esophageal carcinomas. PloS one. 2014; 9:e101010.

11. Choo $\mathrm{Y}$, Isalan M. Advances in zinc finger engineering. Current Opinion in Structural Biology. 2000; 10:411-416.

12. Choo Y, Sanchez-Garcia I, Klug A. In vivo repression by a site-specific DNA-binding protein designed against an oncogenic sequence. Nature. 1994; 372:642-645.

13. Zhang J, Huang JY, Chen YN, Yuan F, Zhang H, Yan FH, Wang MJ, Wang G, Su M, Lu G, Huang Y, Dai H, Ji J, et al.
Whole genome and transcriptome sequencing of matched primary and peritoneal metastatic gastric carcinoma. Sci Rep. 2015; 5:13750.

14. Rink L, Skorobogatko Y, Kossenkov AV, Belinsky MG, Pajak T, Heinrich MC, Blanke CD, von Mehren M, Ochs MF, Eisenberg B, Godwin AK. Gene expression signatures and response to imatinib mesylate in gastrointestinal stromal tumor. Mol Cancer Ther. 2009; 8:2172-2182.

15. Walsh KM. Common genetic variants associated with telomere length confer risk for neuroblastoma and other childhood cancers. Carcinogenesis. 2016; 37.

16. Zhang C, Doherty JA, Burgess S, Hung RJ, Lindström S, Kraft P, Gong J, Amos CI, Sellers TA, Monteiro AN. Genetic determinants of telomere length and risk of common cancers: a Mendelian randomization study. Human Molecular Genetics. 2015; 24:5356-5366.

17. Ojha J, Codd V, Nelson CP, Samani NJ, Smirnov IV, Madsen NR, Hansen HM, de Smith AJ, Bracci PM, Wiencke JK. Genetic variation associated with longer telomere length increases risk of chronic lymphocytic leukemia. Cancer Epidemiology Biomarkers \& Prevention. 2016.

18. Walsh KM, Codd V, Rice T, Nelson CP, Smirnov IV, Mccoy LS, Hansen HM, Elhauge E, Ojha J, Francis SS. Longer genotypically-estimated leukocyte telomere length is associated with increased adult glioma risk. Oncotarget. 2015; 6:42468-42477. doi: 10.18632/oncotarget.6468.

19. Codd V, Nelson CP, Albrecht E, Mangino M, Deelen J, Buxton JL, Hottenga JJ, Fischer K, Esko T, Surakka I, Broer L, Nyholt DR, Mateo Leach I, et al. Identification of seven loci affecting mean telomere length and their association with disease. Nat Genet. 2013; 45:422-427, 427e421-422.

20. Gu Y, Yu C, Miao L, Wang L, Xu C, Xue W, Du J, Yuan H, Dai J, Jin G, Hu Z, Ma H, Shen H. Telomere length, genetic variants and risk of squamous cell carcinoma of the head and neck in Southeast Chinese. Sci Rep. 2016; 6:20675.

21. Du J, Zhu X, Xie C, Dai N, Gu Y, Zhu M, Wang C, Gao Y, Pan F, Ren C, Ji Y, Dai J, Ma H, et al. Telomere length, genetic variants and gastric cancer risk in a Chinese population. Carcinogenesis. 2015; 36:963-970.

22. Risques RA, Vaughan TL, Li X, Odze RD, Blount PL, Ayub K, Gallaher JL, Reid BJ, Rabinovitch PS. Leukocyte telomere length predicts cancer risk in Barrett's esophagus. Cancer Epidemiol Biomarkers Prev. 2007; 16:2649-2655.

23. Xing J, Ajani JA, Chen M, Izzo J, Lin J, Chen Z, Gu J, Wu $X$. Constitutive short telomere length of chromosome $17 \mathrm{p}$ and $12 q$ but not $11 q$ and $2 p$ is associated with an increased risk for esophageal cancer. Cancer prevention research. 2009; 2:459-465.

24. Wentzensen IM, Mirabello L, Pfeiffer RM, Savage SA. The association of telomere length and cancer: a metaanalysis. Cancer Epidemiol Biomarkers Prev. 2011; 20:1238-1250. 
25. Ding H, Yan F, Zhou LL, Ji XH, Gu XN, Tang ZW, Chen RH. Association between previously identified loci affecting telomere length and coronary heart disease (CHD) in Han Chinese population. Clin Interv Aging. 2014; 9:857-861.

26. Wang T, Wang XG, Xu JH, Wu XP, Qiu HL, Yi H, Li WX. Overexpression of the human ZNF300 gene enhances growth and metastasis of cancer cells through activating NF-kB pathway. J Cell Mol Med. 2012; 16:1134-1145.

27. Cheng Y, Geng H, Cheng SH, Liang P, Bai Y, Li J, Srivastava G, Ng MH, Fukagawa T, Wu X, Chan AT, Tao Q. KRAB zinc finger protein ZNF382 is a proapoptotic tumor suppressor that represses multiple oncogenes and is commonly silenced in multiple carcinomas. Cancer Res. 2010; 70:6516-6526.

28. Gao S, Hsieh CL, Zhou J, Shemshedini L. Zinc Finger 280B regulates sGCalpha1 and p53 in prostate cancer cells. PloS one. 2013; 8:e78766.
29. Wang Y, Ye X, Zhou J, Wan Y, Xie H, Deng Y, Yan Y, Li Y, Fan X, Yuan W, Mo X, Wu X. A novel human KRABrelated zinc finger gene ZNF425 inhibits mitogen-activated protein kinase signaling pathway. BMB Rep. 2011; 44:58-63.

30. Thomas RK, Baker AC, Debiasi RM, Winckler W, Laframboise T, Lin WM, Wang M, Feng W, Zander T, MacConaill L, Lee JC, Nicoletti R, Hatton C, et al. Highthroughput oncogene mutation profiling in human cancer. Nat Genet. 2007; 39:347-351.

31. Gabriel S, Ziaugra L, Tabbaa D. SNP genotyping using the Sequenom MassARRAY iPLEX platform. Curr Protoc Hum Genet. 2009; Chapter 2:Unit 212.

32. JM B, DG A. Statistics notes. Bmj. 2000; 320:1468.

33. Shi Y, He L. SHEsis, a powerful software platform for analyses of linkage disequilibrium, haplotype construction, and genetic association at polymorphism loci. Cell Research. 2005; 15:97-98. 